These variations of the order of four times for the blue content are absolute variations, and not shown as a result of the fifty times amplification derived from the masking procedure. Tricolour photometer readings obtained early on any particular night's observation, for a Moon not less than $30^{\circ}$ above the horizon, have so far shown no detectable hue drift over a period of up to $4 \mathrm{~h}$.

It appears that it is only possible to obtain clear colour prints from transparencies (or separation negatives) exposed under relatively 'blue Moon' conditions.

Over the past three years some 30 per cent of ob:ervations showed 'blue Moon' conditions, which do not appear to be statistically less frequent with the approach of the year of the Quiet Sun; for four nights in January 1964 the following were observed, photographed and recorded photoelectrically: January 21 and 26, yellow Moons; January 24 and 28, blue Moons. The photographic record was obtained on a single length of $35 \mathrm{~mm}$ Kodachrome 11 film, processed in one piece by Kodak, Ltd. Tests on standard test objects, including grey scales, for the 'Tripack' colour films used show negligible hue shifts for the full range of exposures used in the telescope.

Stagshead Studios, $159 a$ Ashley Road,

Hale, Altrincham, Cheshire.

'Eastman Kodak Co., Rochester, N.Y., Publ. No. E79.

${ }^{2}$ Wright, W. H., "The Moon as Photographed by Light of Different Colours," Pub. Astro. Soc. Pacific, 41, 125 (1929).

s Miethe, A., and Seegert, B., Astro. Nach.,188, 371 (1911).

\section{On a Possıbility of measuring the Einstein-shift Photoelectrically}

WILLMORE's ${ }^{1}$ method of tracking artificial Earth satellites optically by photoelectric observations seems to be suitable for providing methods of measurement in astronomy with new objectivity and convenience.

The method sets one or more slits in the focal plane of a telescope instead of the reticle, which, as soon as an object appears on them, emit, by a multjplier, an electric signal. Such a system could be appropriate for substituting Repsold's impersonal micrometer; I am now carrying out experimonts along these lines. The main problem with this effort is the improvement of accuracy. Fea and Newton ${ }^{2}$ have also adopted tho method with regard to satellite observations. The accuracy of their device is better than $2 \mathrm{sec}$ of arc. Some of the points of view which led the authors to this figure aro discarded when using a star instead of the fast-moving satellite, and the others, partially, roforring to the equipment, are worth an attempt towards improvement.

Having made the photoelectric methods an equal partner of the conservative astromotric mothods one could make furthor attempts to examine the effect of the deflexion of starlight by the Sun's mass, resulting from the theory of relativity; but in tho past observations made by several astronomers did not accord with theory. Recently, Schmeidler ${ }^{3}$ published a short summary of those efforts.

The use of photoelectric methods should result in faster and surer moasuring methods, and also very faint stars might be recorded because of the sonsitivity of the multiplier tubo being greater than that of the photographic layer.

In principlo the suggested method would measure the places of the shifted stars directly during the eclipse and not thereafter on a photographic plate. Novertheless one should dotermine the places and proper motions of the unshifted stars some months before or (and) later, in the same manner.

For measuring the R.A. of a star it would be sufficient to allow the star to trail across a slit and to rocord the time of crossing. However, the time available is too short to measure the R.A. of all stars of the field, but one might use several slits and multipliers. The positions of the slits have to be calibrated by crossings of fundamental stars. The time and the star signals would be registered on a multichannel recorder.

More difficulties are met in measuring the declinations. One or more slits should be provided parallel to the equator. A slow motion of the slits through the focal plane, that is, an adequate slow motion of the entiro instrument in declination, could replace the motion in hour angle of the device above. This motion having no natural correlation to time we meet the necessity of rocording the momentary position of the slits or the instrument, too. Wo might do this by recording the readings of a level, but since a level should be subject to very slow motions and there is no instrumont good onough to make such registration, we should use, for example, some kind of pendulum device with an olectronic transducer, such devices being capable of measuring fractions of soconds of arc. While measuring the declination in this manner the instrument should follow the epparent movement of the firmament.

Swiss Federal Observatory, Zurich.

' Willmore, A. P., Nature, 182, 1008 (1958).

${ }^{2}$ Fea, K., and Newton, A. C., Planetary and Space Sci., 12, 261 (1964).

${ }^{3}$ Schmeidler, F., Shy and Telescope, 27, 217 (1964).

\section{PHYSICS}

\section{Electron Paramagnetic Resonance in Spinach Chloroplasts at Low Temperature}

Pното-induced electron paramagnetic resonance signals in green plant material have been investigated for many years ${ }^{1-4}$. The description of the parts played by these unpaired electrons in the energy conversion processes of photosynthesis still remains a challenge. This communication describes the initial results of an approach based on the examination of the electron paramagnetic resonance signals at low temperature. It is hoped that the interpretation of the results is simplified in frozen systems by the possibility of excluding mechanisms that proceed with negligible rate in this state, for example, enzymatically catalysed reactions. Thus the observed changes in electron paramagnetic resonance signal intens. ity are expected to occur near the beginning of the energy conversion sequence in photosynthesis.

The olectron paramagnetic resonance spectrometer used in these measurements utilizes a cylindrical $T E_{011}$ mode transmission cavity with balanced barretter mixer detection $^{5}$ and operates at a frequency of about $11 \mathrm{gc} / \mathrm{s}$. Magnetic modulation at a few hundred $\mathrm{c} / \mathrm{s}$ is used for first or second derivative display. The cavity is surrounded by a glass Dewar and its top is cooled with a suitable liquid. Two separate 500-W tungsten projection lamps with water filters, interference filters, and coupled shutters are used to illuminate the sample simultaneously from front and back.

The 'whole' spinach chloroplasts were prepared by the method described by Pon $^{2}$. The samples were run in $3 \mathrm{~mm}$ internal diameter quartz tubes after dilution with buffered sucrose solution to a suitable optical density. The optical transmission coefficients were measured in a flat $3-\mathrm{mm}$ cell at a wave-length of $666 \mathrm{~m} \mu$ with $20 \mathrm{~m} \mu$ bandwidth. The light hitting the surface of the sample in the cavity has a wave-length of $700 \mathrm{~m} \mu$ with about $20 \mathrm{~m} \mu$ band-width and an intensity of $10^{16}$ quanta $/ \mathrm{cm}^{2} / \mathrm{sec}$. The chloroplast concentrations used corresponded to 1 per cent transmission at $666 \mathrm{~m} \mu$ and 10 times this concentration. Neglecting scattering and assuming that the intensity decreases exponentially with sample penetration the 1 per cent transmission results in a maximum light intensity variation of $5: 1$ with the 2 -light system. The 